\title{
Baseline Lymphopenia: A Predictor Of Poor Outcomes In HER2 positive Metastatic Breast Cancer Treated With Trastuzumab
}

This article was published in the following Dove Press journal:

Drug Design, Development and Therapy

\author{
Yi-Qun Che (D)' \\ Yue Zhang' \\ Di Wang $\mathbb{D}^{\prime}$ \\ Hui-Ying Liu ${ }^{2}$ \\ Di Shen (D) \\ Yang Luo $\mathbb{1 D}^{3}$
}

'Department of Clinical Laboratory, National Cancer Center/National Clinical Research Center for Cancer/Cancer Hospital, Chinese Academy of Medical Sciences and Peking Union Medical College, Beijing 10002I, People's Republic of China; ${ }^{2}$ Department of Clinical Laboratory, Beijing Chaoyang District Sanhuan Cancer Hospital, Beijing I00 I22, People's Republic of China; ${ }^{3}$ Department of Medical Oncology, National Cancer Center/National Clinical Research Center for Cancer/Cancer Hospital, Chinese Academy of Medical Sciences and Peking Union Medical College, Beijing I0002I, People's Republic of China
Correspondence: Yang Luo Department of Medical Oncology, National Cancer Center/National Clinical Research Center for Cancer/Cancer Hospital, Chinese Academy of Medical Sciences and Peking Union Medical College, No. 17 Panjiayuan Nanli,

Chaoyang District, Beijing I0002I, People's Republic of China

Tel + 86-10-87788820

$\mathrm{Fax}+86-10-87788820$

Email dryangluo@।63.com
Purpose: Despite selection based on human epidermal growth factor receptor 2 (HER2) overexpression, not all HER2-positive patients benefit from trastuzumab therapy. Recent reports indicate that trastuzumab treatment failure may be associated with immune system dysfunction. We examined the prognostic relevance of the absolute lymphocyte count (ALC) in patients with HER2-positive metastatic breast cancer (MBC) who received trastuzumab combined with chemotherapy.

Methods: Baseline ALC and neutrophil-to-lymphocyte ratio (NLR) data from trastuzumabtreated patients with $\mathrm{MBC}$ were studied retrospectively, and associations between baseline ALC and clinical characteristics evaluated. Kaplan-Meier analysis and the Cox regression hazard model were applied to assess effects on outcomes.

Results: Of a total of 68 patients, 19.1\% (13/68) had baseline ALCs $\leq 1 \mathrm{G} / \mathrm{L}$. Baseline lymphopenia was correlated with increased lactate dehydrogenase (LDH) and higher NLR. In univariate analysis, higher alkaline phosphatase (ALP) was associated with inferior overall survival (OS) $(P=0.001)$; higher LDH was associated with inferior progression-free survival (PFS) $(P=0.045)$ and OS $(P=0.010)$. We did not observe any differences in objective response rate or disease control rate between patients with lymphopenia and those with normal ALC. Importantly, patients with baseline lymphopenia had inferior PFS ( 0.60 years vs 1.17 years, $P=0.000009)$ and OS (1.88 years vs 3.80 years, $P=0.0003)$. In multivariable analysis, significance of ALCs was retained for lymphopenia (PFS: $P=0.0005$; OS: $P=0.016$ ).

Conclusion: Our data indicate that baseline ALC value of $\leq 1 \mathrm{G} / \mathrm{L}$ is a predictor of poor outcomes, but not of response, in patients with MBC treated with trastuzumab.

Keywords: absolute lymphocyte count, metastatic breast cancer, overall survival, progression-free survival

\section{Introduction}

Human epidermal growth factor receptor 2 (HER2) is a member of the epidermal growth factor receptor family that regulates cell differentiation, proliferation, and survival. Approximately $20-30 \%$ of breast cancers overexpress HER2, which is associated with more aggressive tumor behavior and poor prognosis. ${ }^{1,2}$ Treatment with trastuzumab, the first humanized monoclonal antibody developed against HER2, has led to marked improvements in progression-free survival (PFS) and overall survival (OS) for patients with HER2-positive metastatic breast cancer (MBC) as well as in an adjuvant setting. ${ }^{3-5}$ To date, the only validated biomarker for trastuzumab therapy is HER2 amplification. Despite having confirmed HER2-positive 
disease, based on tumor scores of $3+$ by immunohistochemistry (IHC) or identification of amplification by fluorescence in situ hybridization (FISH) testing, ${ }^{6}$ not every individual in this subgroup benefits from trastuzumab treatment. $^{2,3}$ Therefore, clinicians require additional clinical predictors to identify those patients most likely to experience benefit from trastuzumab therapy.

The mechanisms underlying the antitumor activity of trastuzumab are not limited to direct anti-proliferative effects leading to apoptosis of tumor cells, but influences on the immune system, via complement-dependent cytotoxicity (CDC) and antibody-dependent cell-mediated cytotoxicity (ADCC), mediated by immune effector cells. ${ }^{7}$ Trastuzumab may elicit CDC and ADCC upon binding to the HER-2 antigen region on tumor cells, along with the $\mathrm{Fc}$ region of $\mathrm{Fc}$ gamma receptors ( $\mathrm{Fc} \gamma \mathrm{Rs}$ ) expressed by immune cells (e.g., lymphocytes, monocytes, macrophages, eosinophils, and neutrophils). Such an interaction triggers the activation of the corresponding immune cells, resulting in the lysis of trastuzumab-coated tumor cells. ${ }^{8}$ The potential role of immunological mechanisms in the therapeutic efficacy of trastuzumab is supported by several lines of evidence. First, in vitro, tumor cell apoptosis is not observed without the addition of lymphocytes to the culture system. Second, in preclinical models, the antitumor efficacy of trastuzumab is significantly reduced in Fc $\gamma \mathrm{R}$ deficient, compared with wild-type mice. Finally, patient responses to trastuzumab are influenced by the quantity and reactivity of immune effector cells in the tumor microenvironment. $^{9-12}$

Lymphocytes develop in the thymus, circulate in the blood and lymph, and orchestrate immune responses to malignant cells, either by secretion of lymphokines or through direct contact. ${ }^{13}$ Lymphopenia is observed in $20 \%$ of untreated patients with MBC and associated with an increased risk of disease progression and worse longterm survival. ${ }^{14}$ Recently, evidence has emerged supporting the prognostic value of combined scoring systems, based on lymphocyte count, particularly the neutrophilto-lymphocyte ratio (NLR). Elevated NLR is associated with poor prognosis in various cancers, including MBC. ${ }^{15-17}$ Consequently, a number of investigators have inferred that lymphopenia may be associated with immunodeficiency, which could induce antibody resistance; however, the effect of baseline absolute lymphocyte count (ALC) on immune response to trastuzumab treatment remains elusive.
In this context, we aimed to explore the impact of baseline ALC and NLR on response and outcomes following trastuzumab-based treatment of patients with HER2positive MBC.

\section{Materials And Methods Patients}

We recruited 68 patients with HER2-positive MBC who received trastuzumab treatment in the Cancer Hospital, Chinese Academy of Medical Sciences. All patients underwent diagnostic biopsy and/or surgery for a primary or recurred breast tumor before chemotherapy and trastuzumab treatment. All cases were female, with a median age of 51 years. Patient characteristics are summarized in Table 1. All tumors were histologically verified as HER2-amplified according to an IHC score of $3+$ and/or a HER2 FISH ratio of $\geq 2.0 .^{6}$ All patients provided written informed consent. This study was approved by Cancer Hospital, Chinese Academy of Medical Sciences ethics committee, and that it was conducted in accordance with the Declaration of Helsinki. Clinical information for each patient was obtained from medical records or the patients themselves or their clinicians were contacted.

\section{Treatment And Response Evaluation}

Patients received different chemotherapeutic regimens. The most commonly used chemotherapeutic agents were paclitaxel $(32.4 \%)$, vinorelbine $(25.0 \%)$, docetaxel $(25.0 \%)$, gemcitabine $(14.7 \%)$, and capecitabine $(2.9 \%)$. Tumor assessments were performed every two cycles, and disease response was categorized as complete response (CR), partial response (PR), stable disease (SD), or progressive disease (PD), according to the response criteria in solid tumors (RECIST, version 1.1). Objective response rate (ORR) was defined as the presence of either CR or PR. Disease control rate (DCR) was defined as the presence of either CR or PR or SD as best response. PFS was calculated as the time from the initiation of trastuzumab treatment to either disease progression, death from any cause, or last follow-up, while OS was defined as the period from the start of trastuzumab treatment until death from any cause.

\section{Laboratory Assessments}

All included patients underwent baseline routine blood tests, including white blood cell count, with lymphocyte and neutrophil counts, from which the NLR was deduced. Other variables, including alkaline phosphatase (ALP) and lactate dehydrogenase (LDH) levels, were retrieved. Patients were 
Table I Comparison Of Clinical Characteristics Between Lymphopenia And Non-Lymphopenia Group

\begin{tabular}{|c|c|c|c|c|c|c|c|c|}
\hline \multirow[t]{2}{*}{ Characteristic } & & \multicolumn{2}{|c|}{$\begin{array}{l}\text { Lymphopenia } \\
n=13\end{array}$} & \multicolumn{2}{|c|}{$\begin{array}{l}\text { Non-Lymphopenia } \\
n=55\end{array}$} & \multicolumn{2}{|c|}{$\begin{array}{l}\text { Total } \\
n=68\end{array}$} & \multirow[t]{2}{*}{$P$-value } \\
\hline & & $\mathbf{n}$ & $\%$ & $\mathbf{n}$ & $\%$ & $\mathbf{n}$ & $\%$ & \\
\hline Age (years) & Median (range) & \multicolumn{2}{|c|}{$44(31-69)$} & \multicolumn{2}{|c|}{$55(17-74)$} & \multicolumn{2}{|c|}{$51(27-74)$} & 0.813 \\
\hline \multirow[t]{2}{*}{ Menopause status } & Pre- & 4 & 30.8 & 21 & 38.2 & 25 & 36.8 & 0.754 \\
\hline & Post & 9 & 69.2 & 34 & 61.8 & 43 & 63.2 & \\
\hline \multirow[t]{2}{*}{ Karnofsky performance status } & 100 & 2 & 15.4 & 15 & 27.3 & 17 & 25.0 & 0.492 \\
\hline & $70-90$ & 11 & 84.6 & 40 & 72.7 & 51 & 75.0 & \\
\hline \multirow[t]{2}{*}{ Estrogen receptor status } & Positive & 5 & 38.5 & 22 & 40.0 & 27 & 39.7 & 1.000 \\
\hline & Negative & 8 & 61.5 & 33 & 60.0 & 41 & 60.3 & \\
\hline \multirow[t]{2}{*}{ No. of metastatic sites } & 1 & 2 & 15.4 & 22 & 40.0 & 24 & 35.3 & 0.117 \\
\hline & $\geq 2$ & 11 & 84.6 & 33 & 60.0 & 44 & 64.7 & \\
\hline \multirow[t]{2}{*}{ Localization of metastasis } & Liver & 8 & 61.5 & 26 & 47.3 & 34 & 50.0 & 0.539 \\
\hline & Lung & 5 & 38.5 & 21 & 38.2 & 26 & 38.2 & 1.000 \\
\hline LDH (IU/L) & Median (range) & \multicolumn{2}{|c|}{$319(168-543)$} & \multicolumn{2}{|c|}{$217(118-506)$} & \multicolumn{2}{|c|}{$237(118-543)$} & 0.001 \\
\hline ALP (IU/L) & Median (range) & \multicolumn{2}{|c|}{$130(46-344)$} & \multicolumn{2}{|c|}{$97(34-763)$} & \multicolumn{2}{|c|}{$104(34-763)$} & 0.295 \\
\hline NLR & Median (range) & \multicolumn{2}{|c|}{$4.2(1.0-17.0)$} & \multicolumn{2}{|c|}{$2.3(0.6-4.6)$} & \multicolumn{2}{|c|}{$2.7(0.6-17.0)$} & 0.003 \\
\hline
\end{tabular}

Abbreviations: ALP, alkaline phosphatase; LDH, lactate dehydrogenase; NLR, neutrophil-to-lymphocyte ratio.

stratified according to baseline ALC using a cut-off of $1 \mathrm{G} / \mathrm{L}$ based on relevant published reports. ${ }^{18}$ NLR analyses were performed with a threshold of $\geq 3$, according to the results of the meta-analysis by Ethier et al. ${ }^{16}$

\section{Statistical Analysis}

Patients were stratified according to ALC and NLR. Comparisons between variables were performed using the Wilcoxon signed-rank test for numerical variables, and the Fisher exact test for categorical variables. Survival curves were constructed using the Kaplan-Meier method and compared using the log-rank test. The Cox proportional hazards model was applied for multivariate analysis. Candidate prognostic factors with a significance level $\leq 0.10$ in univariate analysis were included in the multivariate analysis. All statistical tests were two-sided, and considered significant when $p$ values were $<0.05$. Statistical analyses were performed using SPSS 22.0 software.

\section{Results}

\section{Comparison With Lymphopenia And Non-Lymphopenia Group According To Baseline ALC}

Median baseline ALC was $1.5 \mathrm{G} / \mathrm{L}$ (range: $0.5-4.1 \mathrm{G} / \mathrm{L}$ ). Thirteen (19.1\%) and $55(80.9 \%)$ patients had baseline
ALC values of $\leq 1 \mathrm{G} / \mathrm{L}$ and $>1 \mathrm{G} / \mathrm{L}$, respectively. The median ages in these two groups (44 [range: 31-69] and 55 [range: 17-74] years, respectively) were not significantly different and neither were menopause status, Karnofsky performance status, hormone receptor status, or number of metastatic sites. There was no remarkable correlation with ALC value according to the presence or the absence of liver or lung metastasis. However, LDH was significantly higher in the group with $\mathrm{ALC} \leq 1 \mathrm{G} / \mathrm{L}$ than in the group with ALC $>1 \mathrm{G} / \mathrm{L}$ (mean $319 \mathrm{IU} / \mathrm{L}$ vs $217 \mathrm{IU} / \mathrm{L} ; P=0.001$ ). Moreover, mean NLR was sharply significant difference between patients with lymphopenia and without lymphopenia $(4.2$ vs $2.3, P=0.003)$ (Table 1).

\section{Effect Of Baseline ALC On Tumor Response}

In the whole group, the ORR was $73.5 \%$ (50 patients), with CR achieved for eight patients (11.8\%). Twelve patients $(17.6 \%$ ) had SD. The PD rate was $8.8 \%$ (six patients), resulting in a DCR of $91.2 \%$ (62 patients). We did not observe any significant difference in ORR $(61.5 \%$ vs $74.5 \%, P=0.49)$ or DCR $(84.6 \%$ vs $94.5 \%, P=0.24)$ between patients with baseline ALC $\leq 1 \mathrm{G} / \mathrm{L}$ and those with ALC > $1 \mathrm{G} / \mathrm{L}$ (Table 2). 
Table 2 Response Evaluation According To Baseline Lymphocyte Count

\begin{tabular}{|c|c|c|c|c|c|c|}
\hline & \multicolumn{2}{|c|}{$\begin{array}{l}\text { Objective Response } \\
\text { n (\%) }\end{array}$} & \multirow[t]{2}{*}{$P$-value } & \multicolumn{2}{|c|}{$\begin{array}{l}\text { Disease Control } \\
\text { n (\%) }\end{array}$} & \multirow[t]{2}{*}{$P$-value } \\
\hline & PD + SD & $C R+P R$ & & PD & $C R+P R+S D$ & \\
\hline $\begin{array}{l}\text { Baseline ALC } \\
\qquad>1 \text { G/L }(n=55) \\
\quad \leq 1 \mathrm{G} / \mathrm{L}(\mathrm{n}=13)\end{array}$ & $\begin{array}{l}14(25.5) \\
5(38.5)\end{array}$ & $\begin{array}{l}4 I(74.5) \\
8(6 \mid .5)\end{array}$ & 0.49 & $\begin{array}{l}3(5.5) \\
2(15.4)\end{array}$ & $\begin{array}{l}52(94.5) \\
\text { II (84.6) }\end{array}$ & 0.24 \\
\hline
\end{tabular}

Abbreviations: PD, progressive disease; SD, stable disease; CR, complete response; PR, partial response; ALC, absolute lymphocyte count.

\section{Influence Of Baseline ALC On Patient Prognosis}

Follow-up data were available for all patients; the median follow-up time was 2.21 years (range: 0.19-8.10 years). During the follow-up period, 61 patients (89.7\%) developed progressive disease, with or without an initial response to trastuzumab therapy. Median PFS was 1.04 years $(95 \% \mathrm{CI}$ : 0.84-1.23 years). Thirty-four patients $(50.0 \%)$ died of recurrence or distant metastasis, resulting in a median OS of 3.58 years (95\% CI: $2.81-4.35$ years). ALP is a hydrolase enzyme that can dephosphorylate various types of molecules, including nucleotides, proteins, and alkaloids. More ALP will be released into the bloodstream during some pathological conditions, including pregnancy, carcinoma, kidney disease, and bone metastasis. Particularly, elevated ALP is frequently reported in bone metastasis in certain malignancies, including osteosarcoma, breast cancer, and prostate cancer. ${ }^{19} \mathrm{LDH}$ is a ubiquitous enzyme that plays a central role in anaerobic glycolysis, as it catalyzes the reversible conversion of pyruvate into lactate. LDH is a well-known marker of tissue damage. Many pathological conditions, including cancer, present with LDH elevation due to acute cell death or necrosis. Moreover, high plasmatic LDH levels influence tumor progression and metastatic spread with a negative impact on outcome in various cancer types. Plasmatic LDH and its variation during first-line treatment predict PFS and OS in MBC, providing independent prognostic information. It would be worthwhile to prospectively evaluate the association between $\mathrm{LDH}$ variation and therapeutic benefit in $\mathrm{MBC}$, and explore how it may affect treatment strategies. ${ }^{20}$ In univariate analysis, higher ALP was associated with inferior OS $(P=0.001)$; similarly, higher LDH was associated with inferior PFS $(P=0.045)$ and OS $(P=0.010)$. The difference was even more significant when we compared the PFS $(P=0.000009)$ and OS $(P=0.0003)$ of patients with $\mathrm{ALC} \leq 1 \mathrm{G} / \mathrm{L}$ and $>1 \mathrm{G} / \mathrm{L}$. Other potentially associated factors (menopause status, hormone receptor expression, liver metastasis, number of metastasis sites, and NLR) were also taken into account (Table 3). Increased LDH and/or ALP were indicators of high tumor load respectively, and they were related to prognosis. Prognosis was analyzed using Kaplan-Meier curves, demonstrating that baseline lymphopenia was significantly associated with inferior PFS (median PFS, 0.597 vs 1.170 years, $P=0.000009$ ) and OS (median OS 1.879 vs 3.803 years, $P=0.0003$ ) in trastuzumab-treated patients (Figure 1). In multivariable analysis, significance was retained for lymphopenia (PFS: hazard ratio [HR]: 0.260, 95\% CI: $0.122-$ 0.552, $P=0.0005$; OS: HR: 0.327, 95\% CI: 0.132-0.812, $P=0.016)$; however, $\mathrm{LDH}$ and ALP did not retain significance (Table 4).

\section{Discussion}

Trastuzumab yields response rates of $8 \%$ to $10 \%$ in advanced, heavily pretreated, and recurrent breast cancer, and its therapeutic efficacy is often enhanced by combination with chemotherapy. ${ }^{1}$ The principal mechanisms of action of trastuzumab include direct growth inhibition, leading to apoptosis of tumor cells, along with CDC and ADCC by natural killer (NK) cells, among others. ${ }^{2}$ Varchetta et al, indicated that the quantity and lytic efficiency of $\mathrm{CD}^{+} 6^{+}$ lymphocytes are major factors influencing ADCC induction by trastuzumab. ${ }^{19}$ Although lymphocytes are fundamental to immunotherapy responses, the potential for ALC to function as a predictor of tumor antigen (TA)-targeted monoclonal antibody efficacy remains unclear. In this study, which evaluated the predictive value of baseline ALC in patients with HER2-positive MBC treated using trastuzumab combined with chemotherapy, we did not identify significant differences in DCR or ORR, using a cut-off value for lymphopenia of $1 \mathrm{G} / \mathrm{L}$; nevertheless, DCR and ORR were lower in patients with lymphopenia than those without. One possible explanation for this is that ADCC represents one mechanism of trastuzumab action, where both inactivation of downstream signal transduction pathways by trastuzumab and 
Table 3 Univariate Analysis Of Prognostic Factors For PFS And OS Of Trastuzumab Therapy In Patients With HER2-Positive MBC ( $\mathrm{n}=68$ )

\begin{tabular}{|c|c|c|c|c|}
\hline \multirow[t]{2}{*}{ Factors } & PFS & \multirow[t]{2}{*}{$P$-value } & os & \multirow[t]{2}{*}{$P$-value } \\
\hline & Median PFS (Years) $95 \% \mathrm{Cl}$ & & Median OS (Years) $95 \% \mathrm{Cl}$ & \\
\hline Menopause & & 0.764 & & 0.939 \\
\hline Pre- & $1.038(0.757-1.320)$ & & 3.471 (1.510-5.432) & \\
\hline Post- & $1.036(0.715-1.356)$ & & $3.740(3.20 \mathrm{I}-4.278)$ & \\
\hline Hormone receptor status & & 0.932 & & 0.999 \\
\hline Positive & $1.07 \mid(0.862-1.280)$ & & $3.422(2.180-4.664)$ & \\
\hline Negative & $0.899(0.569-1.229)$ & & $3.803(2.272-5.333)$ & \\
\hline Liver metastasis & & 0.362 & & 0.159 \\
\hline Yes & 0.921 (0.662-I.I79) & & $3.088(1.970-4.206)$ & \\
\hline No & I.07I (0.872-I.27I) & & $4.096(2.336-5.855)$ & \\
\hline Number of metastasis sites & & 0.672 & & 0.361 \\
\hline Single & I. 107 (0.883-I.330) & & $3.803(3.399-4.207)$ & \\
\hline Multiple & $0.921(0.725-1.116)$ & & $3.422(2.123-4.721)$ & \\
\hline ALC at baseline & & 0.000009 & & 0.0003 \\
\hline$>1 \mathrm{G} / \mathrm{L}$ & I.I $70(0.942-1.397)$ & & $3.803(3.369-4.236)$ & \\
\hline$\leq I \mathrm{G} / \mathrm{L}$ & $0.597(0.382-0.813)$ & & $1.879(1.023-2.736)$ & \\
\hline NLR & & 0.374 & & 0.665 \\
\hline$>3$ & $1.036(0.827-1.245)$ & & $3.47 \mathrm{I}(1.668-5.274)$ & \\
\hline$\leq 3$ & I.07I (0.652-I.49I) & & $3.740(2.894-4.585)$ & \\
\hline LDH at baseline & & 0.045 & & 0.010 \\
\hline$>240 \mathrm{U} / \mathrm{L}$ & $0.858(0.465-\mid .25 I)$ & & $2.334(0.557-4.11 \mathrm{I})$ & \\
\hline$\leq 240 \mathrm{U} / \mathrm{L}$ & I.I 70 (0.888-I.452) & & $3.803(3.396-4.209)$ & \\
\hline ALP at baseline & & 0.064 & & 0.001 \\
\hline$>130 \mathrm{U} / \mathrm{L}$ & $0.858(0.717-0.998)$ & & $2.008(1.333-2.684)$ & \\
\hline$\leq 130 \mathrm{U} / \mathrm{L}$ & I.07I (0.76I-I.38I) & & $3.803(3.373-4.233)$ & \\
\hline
\end{tabular}

Note: Bold values are statistically significant.

Abbreviations: PFS, progression-free survival; OS, overall survival; ALC, absolute lymphocyte count; NLR, neutrophil-to-lymphocyte ratio; LDH, lactate dehydrogenase; ALP, alkaline phosphatase.

chemotherapy could contribute to the response. Another possible explanation is that both sufficient numbers and

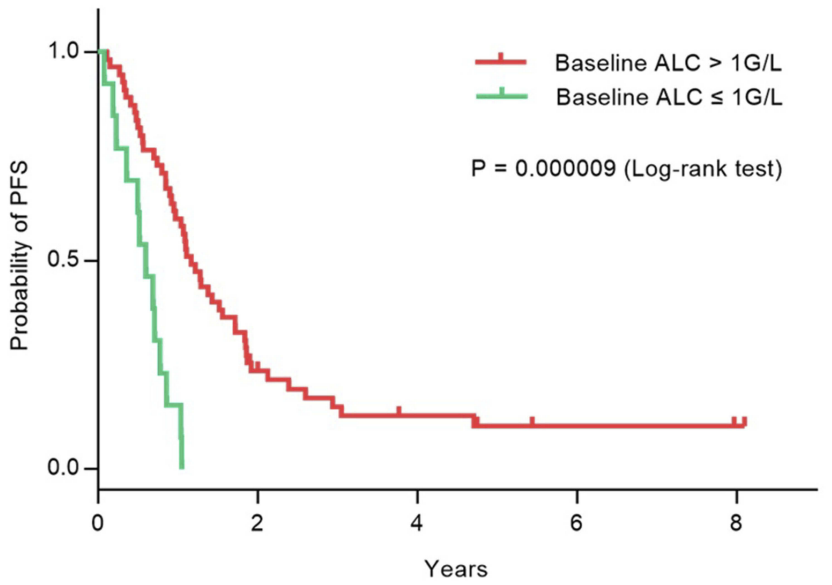

normal function of immune effector cells are essential for the induction of TA-targeted monoclonal antibody-mediated

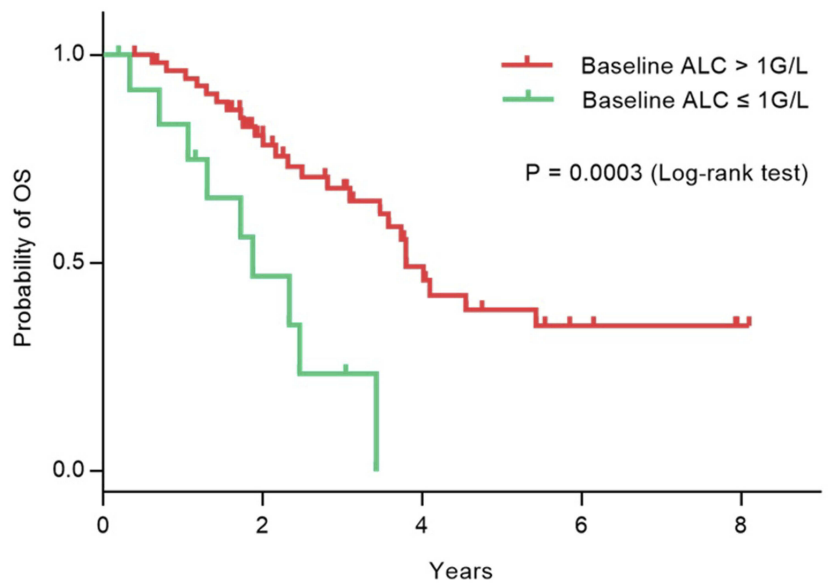

Figure I Kaplan-Meier survival curve for PFS and OS according to baseline ALC (> IG/L vs $\leq$ I G/L) in patients with MBC treated with trastuzumab combined with chemotherapy. 
Table 4 Multivariate Analysis Of PFS And OS $(n=68)$

\begin{tabular}{|c|c|c|c|c|c|}
\hline \multirow[t]{2}{*}{ Factor } & \multirow[t]{2}{*}{ Group } & \multirow{2}{*}{$\begin{array}{l}\text { PFS } \\
\text { HR }(95 \% \mathrm{Cl})\end{array}$} & \multirow[t]{2}{*}{$P$-value } & \multirow{2}{*}{$\begin{array}{l}\text { OS } \\
\text { HR }(95 \% \mathrm{Cl})\end{array}$} & \multirow[t]{2}{*}{$P$-value } \\
\hline & & & & & \\
\hline LDH & $\leq 240 \mathrm{U} / \mathrm{L}$ vs $>240 \mathrm{U} / \mathrm{L}$ & I.175 (0.596-2.314) & 0.642 & $1.327(0.549-3.208)$ & 0.530 \\
\hline ALP & $\leq 130 \mathrm{U} / \mathrm{L}$ vs $>130 \mathrm{U} / \mathrm{L}$ & $1.478(0.666-3.277)$ & 0.337 & $2.736(0.917-7.238)$ & 0.043 \\
\hline Baseline ALC & $\leq 1 \mathrm{G} / \mathrm{L}$ vs $>1 \mathrm{G} / \mathrm{L}$ & $0.260(0.122-0.552)$ & 0.0005 & $0.327(0.132-0.812)$ & 0.016 \\
\hline
\end{tabular}

Note: Bold values are statistically significant.

Abbreviations: PFS, progression-free survival; OS, overall survival; HR, hazard ratio; LDH, lactate dehydrogenase; ALP, alkaline phosphatase; ALC, absolute lymphocyte count.

immune reaction. ${ }^{20-23}$ Tamura reported that Fc $\gamma \mathrm{R} 2 \mathrm{~A}$ polymorphism could predict the pathological response to trastuzumab-based neoadjuvant chemotherapy in patients with early-stage breast cancer, and the objective response to trastuzumab in those with MBC. ${ }^{24}$ More in-depth analyses of peripheral lymphocyte subpopulations may reveal additional information about their predictive potential. Finally, the scale of our study may have been insufficient for the evaluation of minor differences.

Another aim of our study was to evaluate whether baseline ALC and NLR had any prognostic value for patient benefit from trastuzumab. All statistical analysis was conducted using R 3.6.0 (http://www.r-project.org). Hold-out method was used for cross-validation. Samples were randomly assigned to training set and validation set. The sample size and the proportion of outcome event were the same in these two sets. The effects of the factors on OS and PFS in the training set were assessed using a univariate Cox proportional hazards model. We draw the same conclusions after we adopted multiple testing correction and cross-validation to evaluate the robustness of the extracted features. Our findings demonstrate that pretreatment lymphopenia is an independent predictor of poor PFS and OS. Lymphopenia may reflect an impairment of T cell function resulting from immunoparalysis and exhaustion of antitumor lymphocytes, and such deficient lymphocytes may have limited ability to perform antitumor functions during trastuzumab therapy. ${ }^{25}$ The results of this study suggest that patients with lymphopenia are prone to early progression, even those whose breast cancer initially responds to trastuzumab. Our analyses did not identify NLR as a prognostic factor for PFS and OS; however, a Canadian study reported that high NLR $(\geq 3)$ is associated with inferior OS and DFS in patients with breast cancer. ${ }^{16}$ Thus, NLR remains controversial as a prognostic factor for PFS and OS in patients with MBC.

It remains unclear whether lymphopenia is the cause or the consequence of tumor progression. Some host characteristics as well as the tumor burden could result in lymphopenia. In a pooled series, lymphopenia was associated with patient age $(P=0.04)$ and performance status $(P=0.001)$, likely because thymic function in the elderly can contribute to a reduced number of circulating lymphocytes. ${ }^{26}$ Whereas, in this study, we did not observe any association between lymphopenia and age or Karnofsky performance scale status. A possible explanation is that the median age of the patients in our study was only 51 years, and most of them were in the first relapse; hence, the majority of patients had good performance status. However, quantification of total tumor burden at first relapse remains difficult to assess, although it is established that increased LDH is an indicator of high tumor load. In this study, no association was observed between lymphopenia and number of metastases, while lymphopenia was strongly associated with increased LDH, suggesting an association between lymphopenia and high tumor burden.

Although a deeper understanding of the interaction between immunity and combination of trastuzumab and chemotherapy might help optimize or tailor treatment, less is known what is a better option for those patients with baseline lymphopenia. Although there are no reports on this area, lapatinib, a small molecular tyrosine kinase inhibitor, might be available due to its lack the immune activity classically reported with trastuzumab. ${ }^{27}$

\section{Conclusion}

In conclusion, routine blood tests are accessible and fundamental examinations that have long been considered an essential tool assisting cancer diagnosis and treatment. Our results show that baseline lymphopenia may be effective in selecting patients less likely to benefit from trastuzumabbased treatment, despite being validated as HER2-positive. Our study has some limitations, including the small sample size and single-center design; hence, our results require verification by large-scale clinical studies with extensive follow-up. Nevertheless, to the best of our knowledge, this 
study is the first to provide comprehensive insights into associations with ALC in patients with HER2-positive MBC treated with trastuzumab.

\section{Funding}

This work was supported by the Chinese Academy of Medical Sciences Innovation Fund for Medical Sciences (Grant No. 2017-I2M-3-012 and No. 2017-I2M-1-013).

\section{Disclosure}

The authors report no conflicts of interest in this work.

\section{References}

1. Pondé N, Brandão M, El-Hachem G, Werbrouck E, Piccart M. Treatment of advanced HER2-positive breast cancer: 2018 and beyond. Cancer Treat Rev. 2018;67:10-20. doi:10.1016/j.ctrv.2018.04.016

2. Luque-Cabal M, García-Teijido P, Fernández-Pérez Y, SánchezLorenzo L, Palacio-Vázquez I. Mechanisms behind the resistance to trastuzumab in HER2-amplified breast cancer and strategies to overcome it. Clin Med Insights Oncol. 2016;10(Supp11):21-30. doi:10.4137/CMO.S34537

3. Dawood S, Broglio K, Buzdar AU, Hortobagyi GN, Giordano SH. Prognosis of women with metastatic breast cancer by HER2 status and trastuzumab treatment: an institutional-based review. $J$ Clin Oncol. 2010;28(1):92-98. doi:10.1200/JCO.2008.19.9844

4. Perez EA, Romond EH, Suman VJ, et al. Trastuzumab plus adjuvant chemotherapy for human epidermal growth factor receptor 2-positive breast cancer: planned joint analysis of overall survival from NSABP B-31 and NCCTG N9831. J Clin Oncol. 2014;32(33):3744-3752. doi:10.1200/JCO.2014.55.5730

5. Mendes D, Alves C, Afonso N, et al. The benefit of HER2-targeted therapies on overall survival of patients with metastatic HER2-positive breast cancer-a systematic review. Breast Cancer Res. 2015;17 (1):140. doi:10.1186/s13058-015-0648-2

6. Wolff AC, Hammond MEH, Hicks DG, et al. Recommendations for human epidermal growth factor receptor 2 testing in breast cancer: American Society of Clinical Oncology/College of American Pathologists clinical practice guideline update. $J$ Clin Oncol. 2013;31(31):3997-4013. doi:10.1200/JCO.2013.50.9984

7. Muntasell A, Cabo M, Servitja S, et al. Interplay between natural killer cells and Anti-HER2 antibodies: perspectives for breast cancer immunotherapy. Front Immunol. 2017;8:1544. doi:10.3389/fimmu. 2017.01544

8. Boero S, Morabito A, Banelli B, et al. Analysis of in vitro ADCC and clinical response to trastuzumab: possible relevance of FcrRIIIA/ Fc $\gamma$ RIIA gene polymorphisms and HER-2 expression levels on breast cancer cell lines. J Transl Med. 2015;13(1):324. doi:10.1186/s12967015-0680-0

9. Knutson KL, Clynes R, Shreeder B, et al. Improved survival of HER 2 + breast cancer patients treated with trastuzumab and chemotherapy is associated with host antibody immunity against the HER2 intracellular domain. Cancer Res. 2016;76(13):3702-3710. doi:10.1158/0008-5472.CAN-15-3091

10. Shi Y, Fan X, Deng H, et al. Trastuzumab triggers phagocytic killing of high HER2 cancer cells in vitro and in vivo by interaction with Fc $\gamma$ receptors on macrophages. J Immunol. 2015;194(9):4379-4386. doi:10.4049/jimmunol.1402891

11. Mellor JD, Brown MP, Irving HR, Zalcberg JR, Dobrovic A. A critical review of the role of $\mathrm{Fc}$ gamma receptor polymorphisms in the response to monoclonal antibodies in cancer. $J$ Hematol Oncol. 2013;6(1):1. doi:10.1186/1756-8722-6-1
12. Collins DM, O’Donovan N, McGowan PM, O'Sullivan F, Duffy MJ, Crown J. Trastuzumab induces antibody-dependent cell-mediated cytotoxicity (ADCC) in HER-2-non-amplified breast cancer cell lines. Ann Oncol Off J Eur Soc Med Oncol. 2012;23(7):1788-1795. doi:10.1093/annonc/mdr484

13. Gutkin DW, Shurin MR. Clinical evaluation of systemic and local immune responses in cancer: time for integration. Cancer Immunol Immunother. 2014;63(1):45-57. doi:10.1007/s00262-013-1480-0

14. Manuel M, Tredan O, Bachelot T, et al. Lymphopenia combined with low TCR diversity (divpenia) predicts poor overall survival in metastatic breast cancer patients. Oncoimmunology. 2012;1(4):432-440. doi:10.4161/onci.19545

15. Peng B, Wang Y-H, Liu Y-M, Ma L-X. Prognostic significance of the neutrophil to lymphocyte ratio in patients with non-small cell lung cancer: a systemic review and meta-analysis. Int J Clin Exp Med. 2015;8(3):3098-3106. Available from: http://www.ncbi.nlm.nih.gov/ pubmed/26064198. Accessed April 14, 2019.

16. Ethier J-L, Desautels D, Templeton A, Shah PS, Amir E. Prognostic role of neutrophil-to-lymphocyte ratio in breast cancer: a systematic review and meta-analysis. Breast Cancer Res. 2017;19(1):2. doi:10.1186/s13058-016-0794-1

17. Hu P, Shen H, Wang G, Zhang P, Liu Q, Du J. Prognostic significance of systemic inflammation-based lymphocyte- monocyte ratio in patients with lung cancer: based on a large cohort study. El-Deiry WS, ed. PLoS One. 2014;9(9):e108062. doi:10.1371/journal.pone.01 08062

18. Sun R, Champiat S, Dercle L, et al. Baseline lymphopenia should not be used as exclusion criteria in early clinical trials investigating immune checkpoint blockers (PD-1/PD-L1 inhibitors). Eur $J$ Cancer. 2017;84:202-211. doi:10.1016/j.ejca.2017.07.033

19. Varchetta S, Gibelli N, Oliviero B, et al. Elements related to heterogeneity of antibody-dependent cell cytotoxicity in patients under trastuzumab therapy for primary operable breast cancer overexpressing Her2. Cancer Res. 2007;67(24):11991-11999. doi:10.1158/ 0008-5472.CAN-07-2068

20. Kute T, Stehle JR, Ornelles D, Walker N, Delbono O, Vaughn JP. Understanding key assay parameters that affect measurements of trastuzumab-mediated ADCC against Her2 positive breast cancer cells. Oncoimmunology. 2012;1(6):810-821. doi:10.4161/onci.20447

21. Trivedi S, Jie H-B, Ferris RL. Tumor antigen-specific monoclonal antibodies and induction of T-cell immunity. Semin Oncol. 2014;41 (5):678-684. doi:10.1053/j.seminoncol.2014.08.003

22. Alderson KL, Sondel PM. Clinical cancer therapy by NK cells via antibody-dependent cell-mediated cytotoxicity. J Biomed Biotechnol. 2011;2011:379123. doi:10.1155/2011/379123

23. Lee SC, Srivastava RM, López-Albaitero A, Ferrone S, Ferris RL. Natural killer (NK): dendritic cell (DC) cross talk induced by therapeutic monoclonal antibody triggers tumor antigen-specific $\mathrm{T}$ cell immunity. Immunol Res. 2011;50(2-3):248-254. doi:10.1007/s120 26-011-8231-0

24. Tamura K, Shimizu C, Hojo T, et al. Fc $\gamma$ R2A and 3A polymorphisms predict clinical outcome of trastuzumab in both neoadjuvant and metastatic settings in patients with HER2-positive breast cancer. Ann Oncol Off J Eur Soc Med Oncol. 2011;22(6):1302-1307. doi:10.1093/annonc/mdq585

25. Ferris RL, Jaffee EM, Ferrone S. Tumor antigen-targeted, monoclonal antibody-based immunotherapy: clinical response, cellular immunity, and immunoescape. J Clin Oncol. 2010;28(28):4390-4399. doi:10.1200/JCO.2009.27.6360

26. Ferrando-Martínez S, Franco JM, Hernandez A, et al. Thymopoiesis in elderly human is associated with systemic inflammatory status. Age (Dordr). 2009;31(2):87-97. doi:10.1007/s11357-008-9084-x

27. Griguolo G, Pascual T, Dieci MV, Guarneri V, Prat A. Interaction of host immunity with HER2-targeted treatment and tumor heterogeneity in HER2-positive breast cancer. J Immunother Cancer. 2019;7 (1):90. doi:10.1186/s40425-019-0548-6 


\section{Publish your work in this journal}

Drug Design, Development and Therapy is an international, peerreviewed open-access journal that spans the spectrum of drug design and development through to clinical applications. Clinical outcomes, patient safety, and programs for the development and effective, safe, and sustained use of medicines are a feature of the journal, which has also been accepted for indexing on PubMed Central. The manuscript management system is completely online and includes a very quick and fair peer-review system, which is all easy to use. Visit http://www. dovepress.com/testimonials.php to read real quotes from published authors. 